\title{
Rheology of vibrated granular suspensions
}

\author{
Sebastien Kiesgen de Richter ${ }^{1}{ }^{\star}$, Caroline Hanotin $^{2}$, Naima Gaudel $^{1}$, Nicolas Louvet ${ }^{1}$, Philippe Marchal $^{2}$, and Math- \\ ieu Jenny ${ }^{1}$ \\ ${ }^{1}$ Université de Lorraine, LEMTA, CNRS UMR 7563, Vandoeuvre-lès-Nancy, F-54500, France \\ ${ }^{2}$ Université de Lorraine, LRGP, CNRS UMR 7274, Nancy, F-54000, France
}

\begin{abstract}
In this work we investigate in details the flow behaviour of dense vibrated gravitational suspensions. We study the rheology in the stationary state by using a stress imposed rheometer (spectroscopy mechanics) coupled with a vibration cell, we show that applying well-controlled mechanical vibrations allows the control of the suspension viscosity by suppressing the apparent yield stress which is largely the cause of flow jamming. We show that the rheology in the stationary state is controlled by the competition between the reorganization time induced by the flow and the internal reorganization time induced by vibrations. We discuss the influence of particles size, suspending fluid viscosity and vibration parameters and demonstrate that the grains dynamics is controlled by the ratio between the lubrication stress and the granular pressure. This work evidences the major role played by the vibration induced lubrication stress on the liquefaction of vibrated granular suspensions.
\end{abstract}

\section{Introduction}

Industries are looking for tests, correlations or even rheological models for the flow of granular dispersions during transport in relation with some measurable physical features. These correlations and models should, notably, account for the impact of vibrations (amplitude, frequency) on their rheological behavior and structure (rupture, granulation). In practice, vibrations avoid the creation of localized blockages due to the frictional forces between particles and/or with the walls which might result in halting the transport. It is crucial to understand the underlying mechanisms of the blockage and the local effects of applied vibrations to optimize transport. It requires a better understanding of the rheological properties of vibrated granular dispersions, in order to identify the influence of a large number of parameters (size and morphology of particles, viscosity of the interstitial fluid, volume fraction, influence of the applied vibrations, geometrical and confinement effects) on the response of the system. From a more fundamental point of view, studying the influence of vibrations on the flowing behavior of granular dispersions represents a fascinating interplay between the jamming transition in concentrated suspensions and rheology. For instance, understanding the way the particles are organized together and the structure they adopt when vibrations are applied is fundamental to optimize the flow property of this materials. Some crucial common features between dry and fluid saturated monodisperse granular suspensions have recently been evidenced. It has been shown that in both case a visco-plastic $\mu(I)$ rheology describes accurately the flow of such dispersions[1]. The dynamical

\footnotetext{
^e-mail: sebastien.kiesgen@univ-lorraine.fr
}

friction coefficient and therefore the yield stress depends on the dimensionless number $I$, which is the ratio of the internal reorganization time of the dispersion over the typical timescale of the flow. In this respect, the expression of $I$ differs for dry and fluid-saturated flows, and can be interpreted in terms of the relevant time scales controlling grain motions. When vibrations are applied, granular dispersions rheology changes in a drastic way [2-9]. In particular, it modifies the rheology by changing the conditions for flow arrest. One challenge is to generalize the $\mu(I)$ approach when external vibrations control grains reorganizations. In that context, we show in this article that small amplitude vibrations strongly affect the rheology of dense gravitational suspensions. We evidence that the typical reorganization time extracted from mechanical spectroscopy is related to the dynamics of the medium at the grain scale induced by vibrations. This typical time depends on the lubrication Peclet number, $P e_{l u b}$ defines as the ratio between the lubrication and the frictional stresses.

\section{Mechanical spectroscopy}

\subsection{Materials and methods}

We study the viscoelastic behavior of dense gravitational suspensions made of monodisperse glass beads $\left(\rho_{p}=2400 \mathrm{Kg} \cdot \mathrm{m}^{-3}\right)$ immersed in a Newtonian fluid (Water/Polyalkylene glycol mixture, $\rho_{f}=1032 \mathrm{Kg} . \mathrm{m}^{-3}$ ) when both shear and vibrations are applied. In this article, we present results obtained for three different particle diameter $d=49 \pm 3 \mu \mathrm{m}, 100 \pm 8 \mu \mathrm{m}, 335 \pm 15 \mu \mathrm{m}$ and interstitial fluid viscosities ranging from 40 to $160 \mathrm{mPa}$.s. We impose sinusoidal stresses ( rheometer (AR 2000, TA 


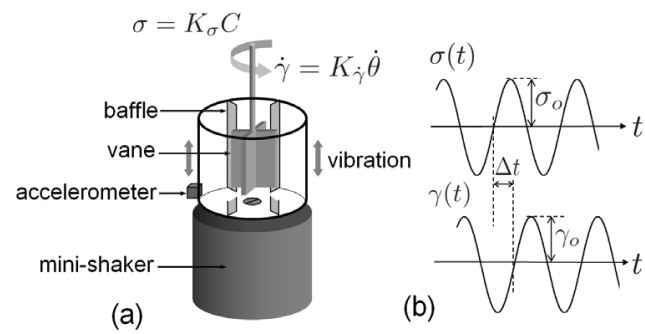

Figure 1. (a) sketch of the experimental setup used for mechanical spectroscopy experiments. (b) Applied sinusoidal shear stess and corresponding measured deformation.

Instruments)) defined by $\sigma(t)=\sigma_{o} \cos (\omega t)$ with $\sigma_{o}$, the oscillation amplitude and $\omega$, the pulsation. The sample was placed into a "powder cell", composed of a cylindrical baffled cup (25 mm radius; $5 \mathrm{~mm}$ baffle width) connected to a vibration shaker(Fig. 1 (a)). Measurements were carried out using a four blade vane device $(10 \mathrm{~mm}$ radius; $30 \mathrm{~mm}$ length)(see hanotin et al. for details). Vertical sinusoidal vibrations are applied with controlled frequency and amplitude. In the following, we define a vibration stress $\sigma_{v}$ defined as the mechanical energy supplied to the sample per unit volume, hence $\sigma_{v}=1 / 2 \rho A^{2}(2 \pi f)^{2}$ with $\rho$ the density of the sample, $A$ and $f$ the amplitude and frequency of vibrations, respectively. The vibration amplitudes and frequencies varies from 30 to $1075 \mu \mathrm{m}$ and from 30 to $60 \mathrm{~Hz}$, respectively.

First, we carried out a stress sweep experiment to ensure experiments are performed in the linear viscoelastic domain. The oscillation amplitude $\sigma_{o}$ is increased from 1 to $1000 \mathrm{~Pa}$, at a fixed frequency $\omega=1 \mathrm{rad} . \mathrm{s}^{-1}$. Moduli are independent of the stress for $\sigma_{o} \leq 50 \mathrm{~Pa}$, and the linear viscoelasticity range is then defined for all $\sigma_{o}$ values lower than $30 \mathrm{~Pa}$. This value changes slightly depending on the experimental parameters, i.e. bead size $(d)$, fluid viscosity $\left(\eta_{f}\right)$ and vibration stress $\left(\sigma_{v}\right)$, but in any case for all samples, a value of $\sigma_{o}=5 \mathrm{~Pa}$ is always located in the linear viscoelasticity domain. For that reason, all frequency sweep tests (variation of $\omega$ between 0.01 and 100 rad.s ${ }^{-1}$, at fixed $\sigma_{o}$ ) were carried out at $\sigma_{o}=5 \mathrm{~Pa}$.

\subsection{Results}

Figure 2 shows the evolution of $G^{\prime}(\omega)$ and $G^{\prime \prime}(\omega)$ for a granular suspension made up of $100 \mu \mathrm{m}$ glass beads for $\eta_{f}=40 \mathrm{mPa} . \mathrm{s}$, with a vibration stress $\sigma_{v}=12 \mathrm{~Pa}(f=$ $40 \mathrm{~Hz}, A=627 \mu \mathrm{m})$. The mechanical spectrum clearly exhibits two distinct regimes. At low frequency, the elastic and viscous moduli display a Maxwell behavior with $G^{\prime} \sim \omega^{2}$ and $G^{\prime \prime} \sim \omega$. In this region, a viscous Newtonian regime is observed in agreement with Hanotin et al(citation). For higher frequencies, the two moduli are equal and increase with $\omega^{1 / 2}$. The transition between both regimes occurs at a critical frequency $\omega_{R}$ corresponding to a critical time $\tau_{R}=1 / \omega_{R}$. We evaluate $\tau_{R}$ by fitting the terminal zone on the basis of a Maxwell model with [10]:

$$
G^{\prime}=G \frac{\omega^{2} \tau_{R}^{2}}{1+\omega^{2} \tau_{R}^{2}} \text { and } G^{\prime \prime}=G \frac{\omega \tau_{R}}{1+\omega^{2} \tau_{R}^{2}}
$$

Where $G$ is the shear modulus (modulus of rigidity).

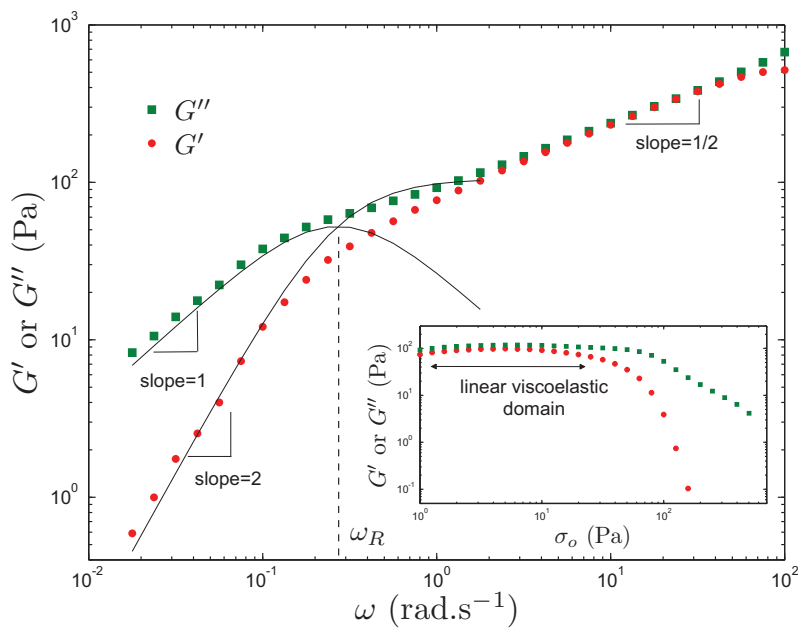

Figure 2. (Color online) Evolution of $G^{\prime}$ and $G^{\prime \prime}$ at a fixed stress amplitude $\sigma_{o}=5 \mathrm{~Pa}$, for $100 \mu \mathrm{m}$ glass beads immersed in a 40 mPa.s Emkarox-water solution (volume fraction $\phi \approx 0.61$ ) and subjected to a vibration stress $\sigma_{v}=12 \mathrm{~Pa}(f=40 \mathrm{~Hz}, A=627$ $\mu \mathrm{m})$. The solid lines correspond to the fit of the terminal zone by Eq. (1) (Maxwell model), with $\tau_{R}=\omega_{R}^{-1}$ the relaxation time. Inset: Stress dependence of the elastic $\left(G^{\prime}\right)$ and viscous moduli $\left(G^{\prime \prime}\right)$, at a fixed pulsation $\omega=1 \mathrm{rad} . \mathrm{s}^{-1}$

From data of Fig. 2, the fit yields $G=105 \mathrm{~Pa}$ and $\tau_{R}=3.7 \mathrm{~s} \Rightarrow \omega_{R}=0.27 \mathrm{rad} . \mathrm{s}^{-1}$. Figure 3 displays the evolution of $\tau_{R}$ as a function of the vibration stress $\sigma_{v}$ for various granular suspensions with three different bead size and fluid viscosity. For any given bead size and fluid viscosity, $\tau_{R}$ decreases when $\sigma_{v}$ increases, and $\tau_{R}$ scales as $\sigma_{v}^{-1 / 2}$. Figure 3 also reveals that $\tau_{R}$ decreases with decreasing bead size, which suggests that rearrangements time are smaller for smaller beads. This is in aggreement with results of [7] which show that the viscosity is controlled by the lubrication Peclet number $P e_{l u b}=\sigma_{l u b} / \sigma_{f}$, such as $\tau_{R} \propto P e_{l u b}^{-1} \propto\left(\eta_{f} A(2 \pi f) / \sigma_{f} d\right)^{-1}$ where $\sigma_{f}$ is the frictional stress (see [7]). According to the same interpretation $\tau_{R}$ should decrease when $\eta_{f}$ increases, which is experimentally confirmed in Fig. 3.

We see from figure 2 that the Maxwell model fails to capture the evolution of the viscoelastic moduli at high frequencies $\left(G^{\prime} \sim G^{\prime \prime} \sim \omega^{1 / 2}\right)$ which suggests the existence of a broad spectrum of relaxation times.

Assuming that the complex modulus $G^{*}$ is the mean of Maxwell modes related to a power law distribution of relaxation times $\rho(\tau)$, it comes that $G^{*}=<\frac{i \omega \tau}{1+i \omega \tau}>$. This relation leads to $G^{\prime}=G^{\prime \prime}$ when the spectrum of relaxation times takes the asymptotic behavior $\rho(\tau) \sim \tau^{-3 / 2}$.

This indicates an inverse square root dependence on frequency similar to that for the anomalous viscous loss 


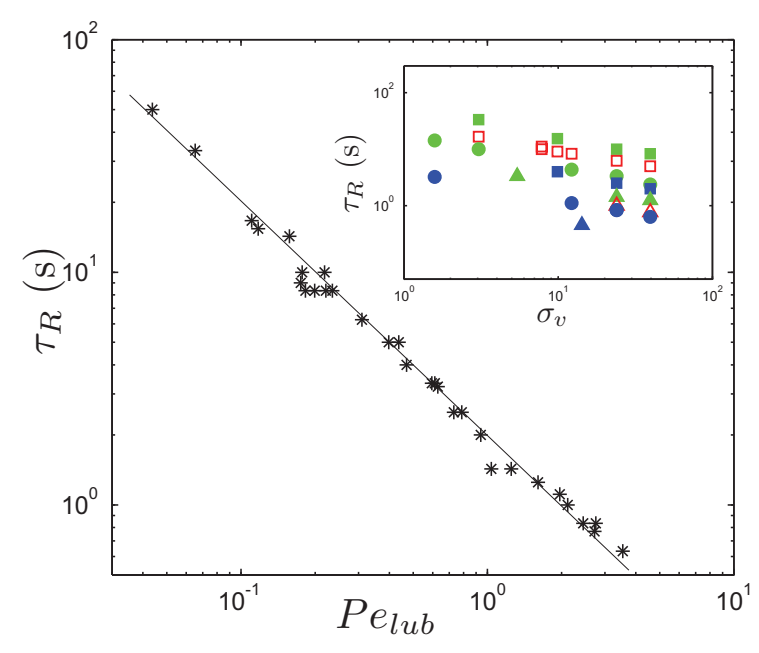

Figure 3. (Color online) (a) Evolution with Peclet number $\left(P e_{l u b}=\frac{\sigma_{l u b}}{\sigma_{f}}\right.$ of the viscoelestic time $\left(\tau_{R}\right)$ extracted from mechanical spectroscopy measurements. Inset: Viscoelastic time $\left(\tau_{R}\right)$ versus vibration stress $\left(\sigma_{v}\right)$, for various values of bead size with diameters $d=335 \mu \mathrm{m}$ (squares), $d=100 \mu \mathrm{m}$ (circles) and $d=49 \mu \mathrm{m}$ (triangles) and for various Emkarox water mixtures with fluid viscosities $\eta_{f}=40 \mathrm{mPa} . \mathrm{s}$ (green), $\eta_{f}=70 \mathrm{mPa} . \mathrm{s}$ (red) and $\eta_{f}=160 \mathrm{mPa} . \mathrm{s}$ (blue).

in emulsions which suggests that the shear strain does not produce affine deformations but some regions slip instead of deforming due to the disorder of the packing.

If the the direction $\cos (\theta)$ of local weak planes with the shear gradient direction varies randomly throughout the material then a broad range of relaxation rates appear with $\tau \sim \tau_{0} \cos ^{-2}(\theta)$ which leads to $G^{*} \sim(i \omega)^{1 / 2}$. Our results suggest that the model proposed by Liu et al.[11] is applicable to report the high frequency behavior of the dynamic shear modulus in vibrated granular suspensions.

\section{Diffusing wave spectroscopy}

\subsection{Materials and methods}

To probe the dynamics at the microscopic scale, we use MSDWS well adapted for sub-microns displacements [12]. The sample is poured into a cylindrical Plexiglas vessel (10 mm diameter; $1 \mathrm{~mm}$ thickness; $50 \mathrm{~mm}$ height), at a height $h=35 \mathrm{~mm}$. $l^{*}$, the average distance traveled by a photon in the sample before its direction becomes randomized approximately equals to $6 d$ in our case with $d$ the bead diameter. We use a He-Ne polarized laser spot of wavelength $\lambda=632.8 \mathrm{~nm}$. The backscattered light is detected by a CCD camera, placed $10 \mathrm{~cm}$ behind the diaphragm. The camera records the different speckle images in 16 bits, with a size of $1392 \times 1040$ pixels. To probe dynamics at long time scales, the amplitude and frequency of vibrations as well as the aperture time of the camera have to be chosen to avoid fast decorrelations of the intensity due to cell vibrations. This condition are achieved by using amplitudes that are small compared to the grain diameters and vibration periods that are short (at least four times lower) with regard to the exposure times of the camera). The technique probes events occurring at long times (typically $>0.1 \mathrm{~s}$ ) by integrating fast variations at short time scales. In our experiments, as the frequency and amplitude used varied from 60 to $160 \mathrm{~Hz}$ and from 4 to $15 \mu \mathrm{m}$, respectively, the samples were subjected to vibration stresses $\sigma_{v}$ lower than 0.1 Pa. Before any data collection, the sample is first vibrated at $f=50 \mathrm{~Hz}$ and $A=400 \mu \mathrm{m}$ during 10 minutes for removing air bubbles. It is then conditioned during five minutes at the desired vibration amplitude and frequency to ensure a "stationary state" where the volume fraction does not change anymore. We measure the intensity correlation function of speckle images by calculating:

$$
\overline{g_{2}\left(t_{0}, t\right)}-1=\frac{\overline{\left\langle I\left(t_{0}+t\right) \cdot I\left(t_{0}\right)\right\rangle_{s p k l}-\left\langle I\left(t_{0}\right)\right\rangle_{s p k l}^{2}}}{\left\langle I\left(t_{0}\right)^{2}\right\rangle_{s p k l}-\left\langle I\left(t_{0}\right)\right\rangle_{s p k l}^{2}}
$$

Where $I\left(t_{0}\right)$ and $I\left(t_{0}+t\right)$ are the intensities of the backscattered light respectively at the reference time $t_{0}$ and at the time $t_{0}+t$ with $t$ the delay time, $\langle\bullet\rangle_{s p k l}$ is the average over different speckles, $\bullet$ is the average over the time origin $t_{0}$. The expression of $g_{2}\left(t_{0}, t\right)-1$ is the one used by Kabla et al. [12] or by Caballero et al. [13].

\subsection{Results}

Figure 4 shows four correlations functions obtained with the same suspension but for different vibration stresses. These function are well fitted by exponential:

$$
\overline{g_{2}\left(t_{0}, t\right)}-1=\beta \exp \left(-2 \gamma \sqrt{6 t / \tau_{D}}\right)
$$

with $\gamma=1.3 \pm 0.3$.

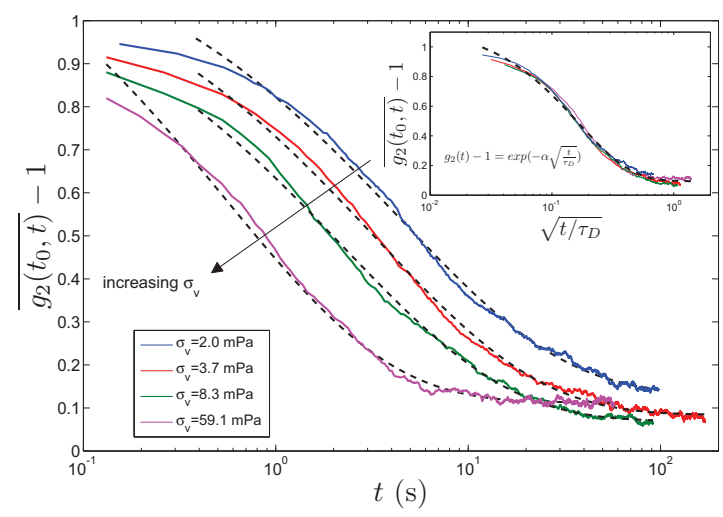

Figure 4. Intensity autocorrelation function $\left(\overline{g_{2}\left(t_{0}, t\right)}-1\right)$ obtained for four different vibrations stresses $\left(\sigma_{v}\right)$, for $200 \mu \mathrm{m}$ glass beads immersed in a $35 \mathrm{mPa}$.s Emkarox-water solution (volume fraction $\phi \cong 0.61$ ). The solid lines represent the experimental data and the dashed lines materialize the fit given by Eq. (3). In the inset, the same data are represented as a function of $\sqrt{t / \tau_{D}}$ with $\tau_{D}$ the decorrelation time extracted from the fit with $\alpha \approx 6$.

Furthermore, the inset of Fig. 4 reveals that all the functions $\overline{g_{2}\left(t_{0}, t\right)}-1$ fall onto a master curve when plotted as a function of the reduced time $\sqrt{t / \tau_{D}}$. It then appears justified to use Eq. (3) for fitting our experimental data 
to extract the decorrelation time $\tau_{D}$. Contrary to Brownian suspensions, the relaxation only results here from externally applied vibrations which induce the rupture of some contacts. The MSDWS technique used in this study probes the frequency of this slipping events (yielding frequency)which cause the displacement of grains and a reorganization of the contact network. Using a phenomenological two-state approach related to the inherent bimodal behavior of chain forces in granular packing ([8]), a given grain can be in two distinct states: a consolidated state(C) that ensures momentum (strong contact network)transport and a mobile state $(\mathrm{M})$ disconnected (weak contact network). The granular medium thus reduces to a two-level system and the typical time extracted from MSDWS measurement can be interpreted as the transition time between states $C \longleftrightarrow M$ induced by external vibrations. Consequently, it is particularly relevant to compare $\tau_{D}$ with $\tau_{R}$ derived from rheological measurements. It clearly appears in Fig. 5 that $\tau_{D}$, as $\tau_{R}$, is controlled by $P e_{l u b}$ and that both times evolve as $1 / P e_{l u b}$. Moreover, the values of $\tau_{D}$ and $\tau_{R}$ overlap over values of $P e_{l u b}$ extending over more than one decade, which means that $\tau_{R}$ completely match with the microscopic measurement of $\tau_{D}$. These results show that the newtonian behavior observed at low shear rates in [7] is direcly related to the internal reorganization of grains due to vibrations. $\tau_{R}$ is linked to the newtonian plateau at low shear viscosity $\eta_{0}=\tau_{R} \sigma_{f} / \gamma_{c}$ which results from the internal dynam- ics of the system induced by vibrations (frequency of yielding events). The critical deformation $\gamma_{c}(\sim 0.82)$ is then related to the breaking of contacts. This approach is in agreement with the recent numerical work of Lin et al. [14] who show that lubrication effects induced by vibrations in dense suspensions can open gaps between particles along chain forces and tunning shear thickening properties.

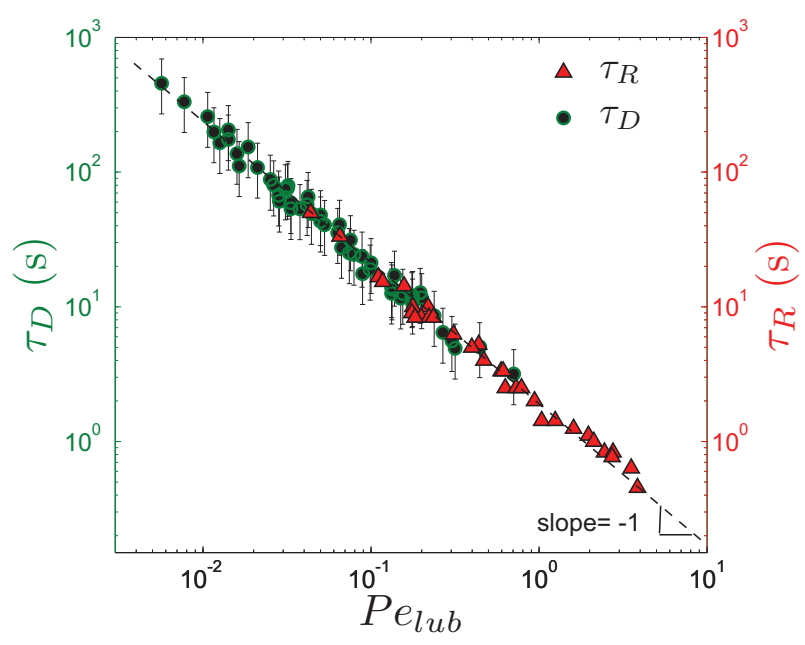

Figure 5. $\tau_{D}$ and $\tau_{R}$ as a function of the Peclet number $\left(P e_{l u b}=\right.$ $\left.\frac{\sigma_{l u b}}{\sigma_{f}} \propto \frac{\eta_{f} A(2 \pi f)}{d \mu \Delta \rho \phi g z}\right)$.

\section{Conclusion}

In this paper, we investigate the dynamics of vibrated granular suspensions by mechanical spectroscopy and multispeckle diffusing wave spectroscopy (MSDWS), with the aim of relating microscopic dynamical mechanisms, at the grain scale, to the rheological behavior of the samples. Results shows that the viscosity of vibrated granular suspensions is controlled by rearrangements dynamics at large time scale related to contact breaking mechanisms. The mean life time of contact $\tau_{D}$ extracted from MSDWS is direcly related to the Maxwell time $\tau_{R}$ obtained by rheological measurements. viscosity are controlled by one relaxation time (larger than the vibration period) related to the flowing behavior of the medium when vibrations are applied which scales as $P e_{l u b}^{-1}$. In the near future, it would be of interest to probe the microscopic dynamics of these systems to visualize in detail how vibrations affect the particle motion at the grain scale. This could be for example achieved by carrying out fast tomography experiments such as those recently developed in synchrotron facilities or by using refractive index matching technics

\section{References}

[1] F. Boyer, E. Guazzelli, O. Pouliquen, Phys. Rev. Lett. 107, 188301 (2011)

[2] G. D’Anna, G. Gremaud, Nature 413, 407 (2001)

[3] G. D’Anna, P. Mayor, A. Barrat, V. Loreto, F. Nori, Nature 424, 909 (2003)

[4] C. Hanotin, P. Marchal, L.J. Michot, C. Baravian, S.K.d. Richter, Soft Matter 9, 9352 (2013)

[5] S.K. de Richter, G. Le Caër, R. Delannay, EPL (Europhysics Letters) 85, 58004 (2009)

[6] S.K. de Richter, C. Hanotin, P. Marchal, S. Leclerc, F. Demeurie, N. Louvet, The European Physical Journal E 38, 1 (2015)

[7] C. Hanotin, S.K. de Richter, P. Marchal, L.J. Michot, C. Baravian, Phys. Rev. Lett. 108, 198301 (2012)

[8] C. Hanotin, S.K.d. Richter, L.J. Michot, P. Marchal, Journal of Rheology (1978-present) 59, 253 (2015)

[9] P. Marchal, L. Choplin, N. Smirani, Journal of Rheology 53, 1 (2009)

[10] J.D. Ferry, Viscoelastic properties of polymers (Wiley New York, 1980)

[11] A.J. Liu, S. Ramaswamy, T. Mason, H. Gang, D. Weitz, Physical review letters 76, 3017 (1996)

[12] A. Kabla, G. Debregeas, Phys. Rev. E. 92, 035501 (2004)

[13] G. Caballero, E. Kolb, A. Lindner, J. Lanuza, E. Clement, J. Phys.: Condens. Matter 17, S2503 (2005)

[14] N.Y. Lin, C. Ness, M.E. Cates, J. Sun, I. Cohen, Proceedings of the National Academy of Sciences $p$. 201608348 (2016) 